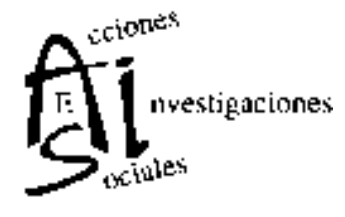

\title{
Evaluación cualitativa de un programa de intervención psicológica con hombres violentos dentro de la pareja
}

\author{
Santiago Boira Sarto, Yolanda lópez del hoyo \\ Y LUCÍA TOMÁS ARAGONÉS \\ DEPARTAMENTO DE PSICOLOGÍA Y SOCIOLOGÍA \\ UNIVERSIDAD DE ZARAGOZA \\ Ana Rosa Gaspar \\ AYUNTAMIENTO DE ZARAGOZA
}

Resumen: La tardía implantación en España de programas de intervención con hombres maltratadores enfatiza la necesidad de desarrollar investigaciones que maximicen la eficacia de los tratamientos. Hasta el momento, han sido escasos los estudios publicados que evalúen las variables relacionadas con la permanencia o el abandono del tratamiento y su eficacia, y todavía más escasos los que han estudiado este tema desde un punto de vista cualitativo. El objetivo del estudio es identificar aspectos clave en los programas de intervención que puedan mejorar la eficacia de los tratamientos para hombres condenados por un delito de violencia de género a los que se les ha suspendido 
o sustituido la ejecución de la pena de prisión por la realización del programa terapéutico. Para ello se ha analizado cualitativamente la información obtenida en dos grupos de discusión formados por los psicólogos que aplicaron el programa y distribuidos según el tipo de terapia, individual o grupal.

Los resultados reflejan la conveniencia de considerar la especificidad de este contexto de intervención en el diseño de los programas y en la evaluación. Deberán mejorarse las estrategias que aumenten la motivación, la alianza terapéutica y la adherencia de los hombres al tratamiento. Además habrá que ampliar las estrategias de evaluación para que ofrezcan una información cualitativamente diferente y permitan una valoración global del impacto del tratamiento y el riesgo para la víctima.

Palabras clave: Violencia contra la pareja, Hombres maltratadores, Programas de intervención por mandato judicial, Estudio cualitativo.

\section{Qualitative assessment of a programme for psychological intervention with men that abuse their partners}

\footnotetext{
Abstract: The late implementation in Spain of intervention programmes for male abusers stresses the need to carry out research that can maximise the effectiveness of such treatment. Up till now, very few studies have been published that assess the variables relating to the continuation or abandonment of the treatment and its efficiency, and there are even fewer studies that examine this subject from a qualitative standpoint. The object of the study is to identify key aspects in the intervention programmes that can enhance the efficiency of the treatment offered to men that have been convicted for a crime involving domestic violence, and who have had their prison sentence suspended or commuted to a programme entailing therapy. With this aim in mind, a qualitative analysis is conducted of the data obtained in two discussion groups formed by psychologists who applied the programme and who are distributed in accordance with the type of therapy -individual or group-oriented- they administered.

The findings reflect the convenience of considering the specifics of this context of intervention in the design of such programmes, along with their assessment. Strategies should be improved to enhance motiva-
} 
tion, and also to strengthen the therapeutic alliance and ensure that the men stick to the treatment. We will also have to broaden our assessment strategies so that they offer data that is qualitatively different and can enable an overall assessment to be made of the impact of the treatment and the risk to the victim.

Keywords: Domestic violence, Male abusers, Intervention programmes by court order, Qualitative study. 


\section{Evaluación cualitativa de un programa de intervención psicológica con hombres violentos dentro de la pareja}

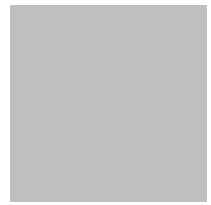

Santiago Boira Sarto Yolanda López del Hoyo Lucía Tomás Aragonés Ana Rosa Gaspar

\section{Introducción}

La aplicación en España de programas para hombres violentos con la pareja es reciente. La primera alternativa estructurada de tratamiento se desarrolló en el año 1996 y hasta el año 2000 sólo se tiene constancia de nueve programas de intervención (Boira, 2010). Este retraso en la implantación ha limitado la publicación de protocolos y propuestas de tratamiento (Arce y Fariña, 2006; Dirección General de Instituciones Penitenciarias, 2005; Echeburúa y Corral, 1998; García, 2005; Quinteros y Carbajosa, 2008; Romero, Rodríguez y Echauri, 2005) y de estudios de evaluación (Boira y Jodrá, en prensa; Echeburúa y Fernández-Montalvo, 2009; Echeburúa, Sarasua, Zubizarreta y Corral, 2009). Con posterioridad a la entrada en vigor de la Ley Orgánica 1/2004, de Medidas de Protección Integral contra la Violencia de Género, el número de programas aumenta debido a la obligación de realizar tratamiento a los condenados por violencia de género (Arce y Fariña, 2010; Expósito y Ruiz, 2010; Lila et al., 2010; Rueda, 2007). Los programas en los que el hombre participa voluntariamente coexisten con los desarrollados dentro de la prisión y en la comunidad pero por mandato judicial. 
Uno de los retos de la intervención con maltratadores en el ámbito de la pareja es identificar los aspectos que puedan mejorar la eficacia de los tratamientos. Las revisiones realizadas a nivel internacional que han evaluado los programas han mostrado resultados discretos (Babcock, Green y Robie, 2004; Feder y Wilson, 2005; Murphy y Ting, 2010; Sartin, Hansen y Huss, 2006; Saunders, 2008). De este modo, algunas de las principales variables incluidas en los procesos de evaluación han sido: a) la orientación teórica del programa; b) la realización obligatoria o voluntaria del mismo; c) el ámbito de aplicación, en la prisión o en la comunidad; y d) el tipo de información utilizada para la evaluación con la inclusión o no del informe de la víctima. De forma general, no se han producido resultados definitivos respecto a la eficacia entre las diferentes modalidades de tratamiento evaluadas. Asimismo, se ha puesto de manifiesto la necesidad de revisar la metodología empleada en la evaluación de los programas y se insiste en encontrar nuevas vías que mejoren tanto la evaluación como la intervención (Gondolf, 2004).

La investigación de la violencia dentro de la pareja y de los programas de rehabilitación para maltratadores ha estado marcada por intensos debates en los últimos años (p.e. Dutton, 2003; Gondolf, 2003; Boira, 2010) y por un estado de permanente revisión y cuestionamiento.

Por otra parte, algunos autores están planteando ampliar las estrategias de evaluación empleadas incorporando medidas de carácter cualitativo que puedan ayudar a mejorar el contexto concreto de intervención (Buchbinder y Eisikovits, 2008). Existen pocas excepciones (Gondolf y Hanneken, 1987; Dobash et al., 2000) en las que se haya estudiado cualitativamente el proceso de intervención con maltratadores.

En España han sido escasos los estudios publicados que evalúen las variables relacionadas con la permanencia o el abandono del tratamiento y su eficacia (Echeburúa y Fernández-Montalvo, 2009; Echeburúa et al., 2009; Boira y Jodrá, 2010) y todavía más escasos los que se cuestionan este tema desde un punto de vista cualitativo y consideran la propia visión de los terapeutas que llevan a cabo las intervenciones.

En este artículo se analiza desde un punto de vista cualitativo la aplicación de un programa de intervención psicológica 
con hombres violentos dentro de la pareja que deben someterse obligatoriamente a la intervención como forma de evitar la ejecución de una pena privativa de libertad.

El objetivo del presente trabajo es identificar aspectos clave que puedan mejorar la eficacia de los tratamientos para hombres condenados por un delito de violencia de género. Para la consecución de este objetivo se realizará un análisis cualitativo de la información proporcionada por los psicólogos que han desarrollado el programa terapéutico.

\section{Método}

\subsection{Participantes}

Los participantes en este estudio fueron terapeutas que aplicaron el programa de intervención, $(n=12 ; 10$ mujeres y 2 hombres) todos ellos psicólogos y especializados en el tratamiento en violencia de género. De ellos, 4 participaron en las condiciones grupales y 8 en las intervenciones individuales. Se aplicó un programa de intervención psicológica de orientación cognitivo conductual a un grupo de 62 hombres condenados por un delito de violencia de género a los que se les ha suspendido o sustituido la ejecución de la pena de prisión.

El programa aplicado es una adaptación del desarrollado por la Secretaría General de Instituciones Penitenciarias para su ejecución dentro de la prisión (Dirección General de Instituciones Penitenciarias, 2005). Los bloques temáticos de los que se compone el tratamiento se muestran en la Tabla 1: 
Tabla 1. Programa de tratamiento (DGIIPP, 2005). Unidades y áreas de intervención.

\begin{tabular}{|ll|}
\hline \multicolumn{1}{|c|}{ Unidades temáticas } & \multicolumn{1}{c|}{ Áreas de trabajo } \\
\hline $\begin{array}{l}\text { Bloque 1. Asunción de la } \\
\text { responsabilidad. }\end{array}$ & $\begin{array}{l}\text { Asunción de la responsabilidad y mecanis- } \\
\text { mos de defensa. } \\
\text { Identificación y expresión de emociones. } \\
\text { Empatía con la víctima. }\end{array}$ \\
$\begin{array}{l}\text { Bloque 2. Control de } \\
\text { emociones. }\end{array}$ & $\begin{array}{l}\text { Distorsiones cognitivas y creencias irracio- } \\
\text { nales. } \\
\text { Ansiedad. } \\
\text { Celos. }\end{array}$ \\
& $\begin{array}{l}\text { Ira y resentimiento. } \\
\text { Bloque 3. Entrenamiento en } \\
\text { habilidades. }\end{array}$ \\
& $\begin{array}{l}\text { Habilidades de relación y comunicación. } \\
\text { Solución de problemas. }\end{array}$ \\
Bloque 4. Cierre. & Educación sexual. \\
& Estilo de vida positivo. \\
& Prevención de recaídas. \\
\hline
\end{tabular}

Los criterios de inclusión que tuvieron que cumplir los hombres agresores para participar en el tratamiento fueron los siguientes: a) hombres condenados por un delito de violencia de género a los que se les ha suspendido o sustituido la ejecución de la pena de prisión e impuesto como medida obligatoria la participación en el programa; y b) consentimiento informado para participar en el estudio. Igualmente, se consideraron los siguientes criterios de exclusión: a) presencia de un trastorno médico o psicológico grave, problemas idiomáticos, intelectuales o de abuso de sustancias que pudieran impedir el correcto desarrollo del protocolo de evaluación y del programa; b) imposibilidad para acudir a las sesiones de intervención; y c) negativa a participar.

Para la aplicación del tratamiento se diseñaron tres grupos, dos en formato grupal ( $A$ y $B$ ) y uno en formato individual. En el formato grupal A, el trabajo terapéutico fue abierto y los contenidos del programa se aplicaron en función de la dinámica del grupo. En el formato grupal B las unidades se presentaron siguiendo un orden estructurado, predefinido en el diseño del 
programa. La frecuencia de las sesiones fue semanal con una duración de 2 horas para los grupos y de aproximadamente una hora para la condición individual.

Los instrumentos seleccionados para la medida de la eficacia de la intervención valoran algunos aspectos que se han considerado esenciales en este tipo de intervención como son la expectativa sobre el tratamiento y la predisposición al cambio (Escala de Evaluación para el Cambio de la Universidad Rhode Island, URICA), variables psicopatológicas (Listado de Síntomas, SCL-90-R) o los aspectos del funcionamiento psicológico relacionados con la dinámica violenta (Inventario de Hostilidad, $\mathrm{BDHI}$; Índice de Reactividad Interpersonal, IRI; Inventario de Pensamientos Distorsionados sobre la Mujer y el Uso de la Violencia).

\subsection{Instrumentos}

La técnica utilizada para el análisis de la información aportada por los terapeutas fue el grupo de discusión. Esta técnica tiene como objetivo la comprensión de una situación en profundidad a través del discurso emergente de las personas participantes (Flick, 1998; Ibáñez, 1989; Krueger, 1991). El objetivo del grupo fue explorar los límites y beneficios de la terapia impartida así como las posibles mejoras para la intervención en este ámbito específico. Las cuestiones y los temas presentados en cada uno de los grupos fueron consensuados por el equipo investigador. La elaboración del guión siguió un esquema semiestructurado y se diseñó ad hoc a partir de la información contenida en el cuestionario que los psicólogos cumplimentan al finalizar la intervención sobre la selección y evaluación de los participantes, la intervención y los resultados del programa.

\subsection{Procedimiento}

Se formaron dos grupos de discusión distribuidos según el tipo de terapia, individual o grupal, este último con independencia de si la intervención fue o no estructurada. El Grupo 1 (terapia individual) estuvo compuesto por 8 terapeutas ( 7 mujeres y 1 hombre) y el Grupo 2 (terapia grupal) por 4 (3 mujeres y 1 hombre). 
Ambos grupos se realizaron un mes después de la finalización del tratamiento con el objetivo fue recuperar la mayor información posible sin que se viese deformada por el efecto del paso del tiempo pero tratando de mantener un espacio temporal entre el debate y la intervención, que permitiese la elaboración de los aspectos terapéuticos centrales. El tiempo empleado para cada uno de los grupos fue aproximadamente de 1 hora y 45 minutos. Se efectuó la grabación en audio y la transcripción literal de la información de cada uno de los grupos. Posteriormente, se procedió al análisis y codificación de los contenidos utilizando el programa de análisis de contenido QSR NVivo (Versión 7). EI análisis de contenido se realizó en base a las dimensiones generales extraídas a partir de las preguntas planteadas en el grupo de discusión. La codificación se realizó utilizando categorías y subcategorías temáticas establecidas a partir de estas dimensiones y a otras nuevas que surgieron a partir del discurso.

Figura 1. Dimensiones generales del análisis.

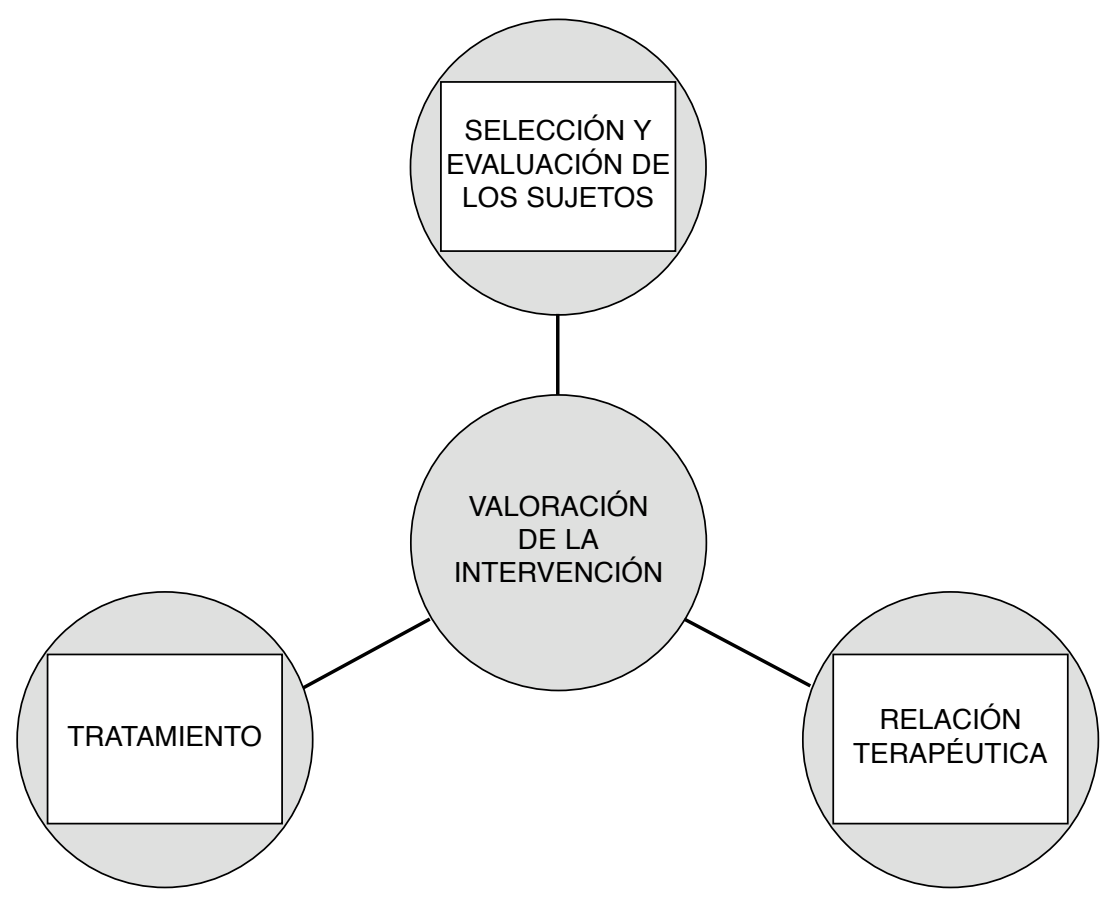




\section{Resultados}

Las categorías emergentes del análisis de contenido y las ideas clave que se han obtenido en las tres dimensiones estudiadas han sido las siguientes:

\subsection{Selección y evaluación de los sujetos}

Las categorías identificadas respecto a la selección y evaluación de los sujetos fueron:

Figura 2. Categorías y análisis de contenido (I).

SELECCIÓN Y EVALUACIÓN DE LOS SUJETOS

- tipología de los agresores

- patología

- ADICCIONES

- VARIABLES SOCIODEMOGRÁFICAS

- EVALUACIÓN

- CRITERIOS DE INCLUSIÓN EXCLUSIÓN

Los terapeutas participantes señalaron la diversidad de características y de perfiles de los hombres sometidos a tratamiento valorando como importante la realización de una evaluación inicial para determinar la posible presencia de patologías y permitir identificar las principales características del hombre. Desde este punto de vista, se manifestó lo siguiente:

"Hay que recoger información para entender la secuencia de la violencia del maltratador, el tipo de violencia que manifiesta y sobre todo entender como piensa (...), su sistema atribucional, su gestión de las relaciones..." (Terapeuta $\mathrm{n}^{\circ} 1$ ).

Se consideró útil la aplicación de pruebas de evaluación antes y después del tratamiento. No obstante, desde un uso clínico de la información, se apuntó el excesivo número de pruebas aplicadas que podría interferir tanto en su correcta 
cumplimentación como en la ya debilitada motivación para comenzar el tratamiento.

Desde el punto de vista del empleo de una entrevista de evaluación inicial, su uso se consideró relevante tanto para la obtención de información como por su valor terapéutico y motivacional. Este hecho fue valorado como muy relevante:

"La entrevista semiestructurada me resulto muy útil utilizada para conocer tanto el desarrollo evolutivo, la situación actual de pareja y de la familia, las relaciones sociales, los posibles antecedentes penales o posibles problemas de maltrato en relaciones anteriores" (Terapeuta $n^{\circ} 3$ ).

"Tener una entrevista previa con estos hombres facilitó la primera sesión de grupo debido a que ya conocen al psicólogo (...), ayuda a explicar, a que te conozcan y a disminuir la resistencia" (Terapeuta $n^{\circ} 9$ ).

"Las entrevistas me llevaron bastante tiempo pero las utilizaba para entrar en la problemática. Aunque las personas negaban el problema como tal, las preguntas me servían para poder indagar y reflexionar sobre cómo habían sido los episodios y tratar de mejorar su toma de conciencia sobre el maltrato" (Terapeuta $\left.\mathrm{n}^{\circ} 2\right)$.

Un tema importante que se puso de manifiesto fue la variedad de situaciones y características de los participantes (tanto desde el punto de vista de la edad, la formación, la ocupación o la nacionalidad). No obstante, no hubo un acuerdo claro en que este hecho pudiera favorecer o dificultar la intervención. Entre las diferencias observadas es necesario destacar las distintas nacionalidades de los participantes en el programa de tratamiento que, en muchos casos, implicó la existencia de diferentes patrones culturales y la presencia de dificultades con la comprensión del idioma:

"Yo en la entrevista la mayor dificultad que encontré fue con el idioma, motivación no tenían ninguna, venían porque tenían que venir (...) a la hora de hablar con ellos con el idioma sí que había algún problema, algunas cosas no entendían" (Terapeuta $\left.n^{\circ} 2\right)$.

"A nivel de diferencia del entorno sociocultural se veían muchas cosas, lo que es el entorno familiar, la valoración de la mujer y la violencia" (Terapeuta $\left.n^{\circ} 1\right)$. 
En este sentido, tanto en el caso de presencia de una psicopatología grave como por la dificultad de comprensión del idioma se valoró positivamente la determinación de unos criterios de exclusión del programa claros. Se consideró importante la evaluación previa que discriminara aquellos sujetos que no debían incorporarse al proceso de tratamiento.

Finalmente, pese a que se consideró adecuada la elección de las pruebas y cuestionarios de evaluación, se señaló que algunos de los cambios observados durante en proceso de intervención no se recogieron adecuadamente con la evaluación propuesta.

\subsection{Relación terapéutica}

Las categorías emergentes en relación con la relación terapéutica han sido las siguientes:

Figura 3. Categorías y análisis de contenido (II).

$$
\text { RELACIÓN TERAPÉUTICA }
$$

- encuadre

- ALIANZA TERAPÉUTICA

- RESISTENCIA DEL PENADO

- MOTIVACIÓN

- RESPUESTA DEL TERAPEUTA

- PENADOS PROBLEMÁTICOS

Los terapeutas consideraron fundamental el encuadre de la intervención con el participante para que ésta se desarrolle adecuadamente (carácter obligatorio de la intervención, el secreto, los objetivos terapéuticos, las normas, etc.).

"Informar en qué iba a consistir el grupo, cuáles son las responsabilidades, qué se le va a exigir al participante (...), cuánto dura el grupo, el horario, la asistencia. En el grupo fue bien 
encuadrar, el encuadre fue claro y firme aunque luego se flexibilizó en algún momento (...) El hecho de decirles a los hombres que se les podía llamar entre sesión y sesión para venir, que tendrían que venir y, si no, se informaría al Servicio Social Penitenciario y al juez..." (Terapeuta $\left.n^{\circ} 8\right)$.

Hay que tener en cuenta que en este tipo de intervención se solapan el marco terapéutico con el de ejecución de una medida penal. El encuadre de la terapia debe considerar los dos planos $y$, en este sentido, se valoró como positivo el establecimiento de unos límites claros entre lo "legal" y lo "psicológico". Respecto al encuadre, y como comenta la terapeuta $n^{\circ} 6$ :

"EI mayor problema que hay es que ellos no se consideran culpables de lo que han hecho y no necesitan tratamiento".

Entre las características más habituales que lo terapeutas indicaron respecto a los hombres participantes en el programa se señaló la negación del maltrato, la ausencia de conciencia sobre el impacto de su comportamiento violento y su baja motivación para el cambio. Algunos terapeutas manifestaron que una parte de los hombres negaban directamente el haber ejercido ningún comportamiento de malos tratos:

"Negar los hechos o era para ellos normal, ellos tampoco tenían sentimiento de culpabilidad" (Terapeuta $n^{\circ} 5$ ).

En otros casos se comentó que aunque se reconocía el hecho puntual, el hombre no le otorgaba la importancia suficiente. No se veía capaz de tener presente la secuencia del maltrato y del auténtico significado de la violencia situándose en una posición de víctima:

"Aunque el penado ve mal ese momento, el cree que se están portando mucho peor con él, ve un desfase muy grande entre lo que él ha hecho y lo que le están haciendo a él" (Terapeuta $\left.n^{\circ} 1\right)$.

En cualquiera de los casos, se valoró como esencial el establecimiento de estrategias para mejorar la alianza terapéutica y la adherencia al tratamiento:

"Se trata de abordar cómo podemos intervenir en situaciones donde no sólo no hay conciencia de problema sino que el otro no está por la intervención: ¿cómo hacer un encuadre y fomentar una alianza terapéutica en situaciones muy difíciles?" (Terapeuta $\left.n^{\circ} 11\right)$. 
En este contexto, la actitud del terapeuta puede resultar muy significativa. Tanto desde el punto de vista de la terapia individual como grupal, algunos terapeutas manifestaron su dificultad para manejar la intervención en una situación en la que prevalece la resistencia y las respuestas hostiles del penado. Aunque difícil de conseguir, hay bastante acuerdo en admitir que una actitud empática puede resultar relevante en la consecución de los objetivos de la intervención:

"En el desarrollo del tratamiento ha resultado fundamental la empatía y la utilización de un lenguaje muy cuidado, fundamentalmente descriptivo" (Terapeuta $\left.n^{\circ} 1\right)$.

"A mi me resulto muy útil mantener una actitud empática, para trasmitirles confianza y a través de una escucha activa proporcionales ayuda en sus necesidades de desahogo, sobre todo en las primeras citas. A pesar de estas primeras resistencias conseguí una implicación de los participantes, mostrándose más motivados y receptivos al tratamiento al planteárselo como una oportunidad de crecimiento personal (Terapeuta $n^{\circ} 3$ ).

Por último, se valoró positivamente la presencia de dos terapeutas en los grupos no pareciendo significativo el sexo de los mismos.

\subsection{Tratamiento}

Por último, las categorías estudiadas en esta dimensión han sido:

Figura 4. Categorías y análisis de contenido (III).

TRATAMIENTO

- OBLIGATORIEDAD

- CONTEXTO DE LA INTERVENCIÓN

- Clima gRUPAL

- TIPO DE PROGRAMA (INDIVIDUAL/GRUPAL)

- CONTENIDO/ORIENTACIÓN PROGRAMA

- MATERIALES 
Globalmente, y teniendo en cuanta las limitaciones del marco en que este programa es aplicado, el desarrollo de la intervención tanto grupal como individual se ha considerado adecuada. Algunas de las expresiones en este sentido han sido las siguientes:

"Creo que el programa resulta útil para los participantes, pero creo que no es suficiente (...) Creo que la finalización de la terapia debería estar condicionada al logro de objetivos concretos y que los participantes deberían saberlo“ (Terapeuta $n^{\circ} 1$ ).

"Creo que es un programa que requiere mucho tiempo de trabajo, dada la complejidad y el arraigo de las creencias distorsionadas de esta población (...) Por otro lado, también he tenido la oportunidad de tratar personas motivadas para la intervención y en las que he visto una evolución satisfactoria (Terapeuta $n^{\circ} 4$ ).

"A mi parecer el tratamiento resulta un instrumento útil ya que ayuda al agresor a ser consciente de su problema, a asumir su responsabilidad en los episodios de violencia, a conocer y controlar sus propios pensamientos y emociones, y a aumentar el repertorio de habilidades de comunicación y de solución de problemas (Terapeuta $\mathrm{n}^{\circ} 3$ ).

No obstante, se encuentran diferencias en la percepción de los terapeutas en la implicación y el aprovechamiento de los diferentes participantes. Desde el punto de vista del cambio, una de las terapeutas comenta:

"Con algunos se llegó pero no se remató, con otro se hubiera llegado (en el caso de que la terapia hubiera continuado) y yo creo que otro no y además están señalados" (Terapeuta $n^{\circ} 8$ ).

En cuanto al modo en que la intervención se desarrolla se plantearon dos puntos importantes en la discusión: a) la identificación del tipo de estrategia que puede ser mas eficaz para promover el cambio y la asunción de la responsabilidad del comportamiento violento (más confrontadora, más nutritiva, más pedagógica...) y b) la valoración del orden en que se van proponiendo los diferentes objetivos de la terapia. En este sentido, algunos de los puntos de vista que se plantearon fueron los siguientes:

De cara a que el participante asuma su responsabilidad y tenga una postura abierta respecto en la terapia: "yo no sé si el conflicto es nutrir o confrontar al maltratador, para mí va bien 
señalar y, respecto a la asunción de responsabilidad, exigir responsabilidades mucho más pequeñas (Terapeuta $\mathrm{n}^{\circ} 8$ ).

En este sentido, y en relación con el tema de la ausencia de conciencia de actitudes de carácter machista o del ejercicio de un tipo de violencia más sutil y psicológica, esta terapeuta continua: "para mi es como una carrera de fondo, es como algo que yo veo que me parece obvio pero el otro no lo ve y creo que hay que señalarlo tantas veces como el otro necesita" (Terapeuta $n^{\circ} 8$ ).

Otro de los terapeutas, sin embargo, afirma que es imprescindible que el penado asuma al menos parte de la responsabilidad sobre su comportamiento antes de abordar otros contenidos del programa. Lo expresa de la siguiente manera:

"Mi gran preocupación siempre ha sido, si pasamos a otro tipo de bloque como habilidades sociales o habilidades de comunicación sin habernos asegurado de que ha habido cambios en la asunción de responsabilidad podemos estar enseñando a ser maltratadores más sutiles" (Terapeuta $\mathrm{n}^{\circ} 7$ ).

En este contexto, algunas propuestas se encaminan a la posibilidad de combinar dentro del programa la intervención grupal y la individual. Una de las terapeutas propone que:

"el programa de intervención ideal podría ser la aplicación de un tratamiento individual, ajustado a las necesidades especificas de cada persona, intercalado con sesiones grupales (de unos 8-10 sujetos), en las que confrontarse con otros sujetos en su misma o parecida situación (Terapeuta $n^{\circ} 7$ ).

Así, hay bastante acuerdo en la posibilidad de flexibilizar la aplicación de los bloques de contenido del programa en función de las características de los participantes tratando de adecuar los contenidos de la terapia al tipo de agresor. Asimismo, se propone incluir un bloque específico de género y violencia.

Respecto al contexto en que este tipo de terapia ser realiza, se indica la importancia de estudiar el modo en que puede afectar a la intervención el contexto terapéutico (medio penitenciario, servicios sociales, ONGs, etc.). Los terapeutas participantes propusieron como óptima la combinación de la intervención individual con la grupal.

Por otra parte, en general se plantea que el número de sesiones del programa se consideró escaso y el número de participantes (15/16 por grupo) excesivo. Asimismo, la frecuencia 
semanal de sesiones se consideró adecuada para esta población.

\section{Discusión}

El análisis cualitativo pone de manifiesto la repercusión que la baja motivación para el cambio representa tanto en la intervención como en la evaluación. Las dificultades metodológicas para evaluar los programas vienen determinadas en muchas ocasiones por un contexto de intervención definido por la negación del comportamiento violento, la baja motivación para el cambio y por la obligatoriedad de realizar el tratamiento (Enosh, 2008). Esta situación se acompaña a menudo de la desconfianza en el sistema penal y de una percepción de desprotección jurídica por parte del maltratador. Digard (2010) estudió este aspecto con un grupo de agresores sexuales debido a su importancia en la eficacia de la intervención y en el futuro comportamiento del agresor.

Por otra parte, en futuros estudios de evaluación de programas dirigidos a maltratadores debe plantearse el marco de intervención como variable relevante (Bowen, 2010) y considerar que los resultados pueden diferir en función de aspectos como el tipo delictivo, la obligatoriedad, la motivación o las expectativas puestas en el tratamiento. En este sentido, deberán mejorarse las estrategias que aumenten la motivación, la alianza terapéutica y la adherencia de los hombres al tratamiento (Dia, Simmons, Oliver y Cooper, 2009; Maiuro y Murphy, 2009; Musser, Semiatin, Taft y Murphy, 2008; Taft y Murphy, 2007) en un contexto que inicialmente puede quedar definido como no terapéutico (Cirillo, 1994).

De la interpretación de los datos obtenidos se deducen algunos temas de interés para la evaluación: a) la resistencia al tratamiento puede provocar que el sujeto falsee las pruebas; b) la necesidad de revisar la utilización de cuestionarios de autoinforme como estrategia principal en la evaluación del cambio; y c) la importancia de usar instrumentos de evaluación cada vez más específicos al contexto de intervención con maltratadores.

Respecto al futuro, en el diseño de los programas será necesario valorar el tipo de intervención propuesta en función de la 
predisposición al cambio del maltratador, de sus características diferenciales y del contexto de intervención. De la misma forma, habrá que ampliar las estrategias de evaluación para que ofrezcan una información cualitativamente diferente y permitan una valoración más global del impacto de los tratamientos y del riesgo para la victima (Buchbinder y Eisikovits, 2008; Shamai y Buchbinder, 2010).

\section{Referencias Bibliográficas}

ARCE, R. y FARIÑA, F. (2006). "Programa Galicia de reeducación para maltratadores de género". Anuario de Psicología Jurídica, 16, 41-64.

ARCE, R. y FARIÑA, F. (2010). "Diseño e implementación del Programa Galicia de reeducación de maltratadores: una respuesta psicosocial a una necesidad social y penitenciaria". Intervención Psicosocial, 19, 153-166.

BABCOCK, J.C., GREEN, C.E. y ROBIE, C. (2004). "Does batterers' treatment work? A meta-analytic review of domestic violence treatment”. Clinical Psychology Review, 23, 10231053.

BOIRA, S. (2010). Hombres maltratadores. Historias de violencia masculina. Zaragoza: Prensas Universitarias de Zaragoza.

BOIRA, S. y JODRÁ, P. (2010). "Psicopatología, características de la violencia y abandonos en programas para hombres violentos con la pareja: resultados en un dispositivo de intervención". Psicothema, 22, 593-599.

BOWEN, E. (2010). "Therapeutic environment and outcomes in a U.K. domestic violence perpetrator program". Small Group Research, 41, 198-220.

BUCHBINDER, E. y EISIKOVITS, Z. (2008). "Doing treatment: Batterers' experience of intervention". Children and Youth Services Review, 30, 616-630.

CIRILLO, S. (1994). El cambio en los contextos no terapéuticos. Barcelona: Paidós.

DIA, D.A., SIMMONS, C., OLIVER, M., y COOPER, R.L. (2009). "Motivational interviewing for intimate partner violence". En P. Lehmann y C.A. Simmons (Eds.), Strengths based batterers 
intervention: a new paradigm in ending family violence (pp. 87-112). New York: Springer Publishing.

DIGARD, L. (2010). "When legitimacy is denied: Offender perceptions of the prison recall system". Probation Journal, 57, 43-61.

DIRECCIÓN GENERAL DE INSTITUCIONES PENITENCIARIAS. (2005). Programa de tratamiento en prisión para agresores en el ámbito familiar. Grupo de trabajo sobre violencia de género. Madrid: Secretaría General Técnica. Ministerio del Interior.

DOBASH, R.E., DOBASH, R.P., CAVANAGH, K. y LEWIS, R. (2000). Changing Violent Men. Thousand Oaks, CA: Sage.

DUTTON, D.G. (2003). "MCMI results for batterers: A response to Gondolf". Journal of Family Violence, 18(4), 253-255.

ECHEBURÚA, E. y CORRAL, P. (1998). Manual de violencia familiar. Madrid: S. XXI.

ECHEBURÚA, E. y FERNÁNDEZ-MONTALVO, J. (2009). "Evaluación de un programa de tratamiento en prisión de hombres condenados por violencia grave contra la pareja". International Journal of Clinical and Health Psychology, 9, 5-20.

ECHEBURÚA, E., SARASUA, B., ZUBIZARRETA, I. y CORRAL, P. (2009). "Evaluación de la eficacia de un tratamiento cognitivo-conductual para hombres violentos contra la pareja en un marco comunitario: una experiencia de 10 años (19972007)". International Journal of Clinical and Health Psychology, 9, 199-217.

ENOSH, G. (2008). "Resistance to evaluation in batterers' programs in Israel. Children and Youth Services Review", 30, 647-653.

EXPÓSITO, F. y RUIZ, S. (2010). "Reeducación de Maltratadores: una experiencia de intervención desde la perspectiva de género". Intervención Psicosocial, 19, 145-151.

FEDER, L. y WILSON, D.B. (2005). "A meta-analytic review of court-mandated batterer intervention programs: Can courts affect abusers' behavior?". Journal of Experimental Criminology, 1, 239-262.

FLICK, U. (1998). An introduction to qualitative research. Londres: Sage.

GARCÍA, Y. (2005). Programa de tratamiento para hombres con relaciones familiares violentas. Logroño: Consejería de 
Juventud, Familia y Servicios Sociales. Gobierno de La Rioja.

GONDOLF, E.W. (2003). "MCMI Results for Batterers: Gondolf Replies to Dutton's Response". Journal of Family Violence, 18(6), 387-389.

GONDOLF, E.W. (2004). "Evaluating batterer counseling programs: A difficult task showing some effects and implication". Aggression and Violent Behavior, 9, 605-631.

GONDOLF, E. W. y HANNEKEN, J. (1987). "The gender warrior: Reformed batterers on abuse, treatment, and change". Journal of Family Violence, 2, 177-191.

IBÁÑEZ, J. (1989). "Cómo se realiza una investigación mediante grupos de discusión". En M. García, J. Ibáñez y F. Alvira (Eds.), El análisis de la realidad social: métodos y técnicas de investigación (pp. 489-501). Madrid: Alianza Editorial.

KRUEGER, R.A. (1991). "El grupo de discusión: guía práctica para la investigación aplicada". Madrid: Pirámide.

LILA, M., CATALÁ, A., CONCHELL, R., GARCÍA, A., LORENZO, M.V., PEDRÓN, V. y TERREROS, E. (2010). "Una experiencia de investigación, formación e intervención con hombres penados por violencia contra la mujer en la Universidad de Valencia: Programa Contexto". Intervención Psicosocial, 19, 167-179.

MAIURO, R.D. y MURPHY, C.M. (Eds.). (2009). Motivational interviewing and stages of change in intimate partner violence. Nueva York: Springer.

MURPHY, C.M. y TING, L.A. (2010). "Interventions for perpetrators of intimate partner violence: a review of efficacy research and recent trends". Partner Abuse, 1, 26-44.

MUSSER, P.H., SEMIATIN, J.N., TAFT, C.T. y MURPHY, C.M. (2008). "Motivational interviewing as a pregroup intervention for partner-violent men". Violence and Victims, 23, 539-557.

QUINTEROS, A. y CARBAJOSA, V. (2008). Hombres maltratadores: tratamiento psicológico de agresores. Madrid: Grupo 5.

ROMERO, J., RODRíGUEZ, M.J. y ECHAURI, J.A. (2005). "Teoría y descripción de la violencia doméstica. Programa terapéutico para maltratadores del ámbito familiar en el centro penitenciario de Pamplona. "Anuario de Psicología Jurídica, $15,67-91$. 
RUEDA, M.A. (2007). Los programas y/o tratamientos de los agresores en supuestos de violencia de género: ¿Una alternativa eficaz a la pena de prisión? Madrid: Librería-Editorial Dykinson.

SARTIN, R.M., HANSEN, D.J. y HUSS, M.T. (2006). "Domestic violence treatment response and recidivism: $A$ review and implications for the study of family violence". Journal of Aggression and Violent Behavior, 11, 425-440.

SAUNDERS, D.G. (2008). "Group interventions for men who batter: A summary of program descriptions and research". Violence and Victims, 23, 156-172.

SHAMAI, M. y BUCHBINDER E. (2010). "Control of the self: partner-violent men's experience of therapy". Journal of Interpersonal Violence, 25, 1338-1362.

TAFT, C.T. y MURPHY, C.M. (2007). "The working alliance in intervention for partner violence perpetrators: recent research and theory". Journal of Family Violence, 22, 11-18. 